\title{
Hepatoid esophagogastric adenocarcinoma and tumoral heterogeneity: a case report
}

\author{
Tiago Biachi de Castria ${ }^{1,2}$, Laura Tang ${ }^{2,3}$, Marcello Moro Queiroz ${ }^{1}$, Beatriz Mendes Awni ${ }^{1}$, \\ Viktoriya Paroder ${ }^{2,3}$, Ali Shamseddine ${ }^{4}$, Giovanni Mendonca Bariani ${ }^{1}$, Deborah Mukherji ${ }^{4}$, \\ Charbel F. Matar ${ }^{4}$, Gustavo dos Santos Fernandes ${ }^{1}$, Ashwaq El-Olayan ${ }^{5}$, Fouad Sabatin ${ }^{5}$, \\ Rawad Elias $^{6}$, Ranju Gupta ${ }^{7}$, Yelena Y. Janjigian ${ }^{2,3}$, Ghassan K. Abou-Alfa ${ }^{2,3}$ \\ ${ }^{1}$ Centro de Oncologia, Hospital Sírio-Libanês, São Paulo, SP, Brasil; ${ }^{2}$ Memorial Sloan Kettering Cancer Center, New York, NY, USA; ${ }^{3}$ Weill Medical \\ College at Cornell University, New York, NY, USA; ${ }^{4}$ American University of Beirut, Beirut, Lebanon; ${ }^{5}$ National Guard Hospital, King Abdulaziz \\ Medical City, Riyadh, Kingdom of Saudi Arabia; ${ }^{6}$ Hartford HealthCare Cancer Institute at Hartford Hospital, Hartford, CT, USA; ${ }^{7}$ Lehigh Valley \\ Cancer Institute, Bethlehem, PA, USA \\ Correspondence to: Ghassan K. Abou-Alfa. Memorial Sloan Kettering Cancer Center, 300 East 66th Street, New York, NY 10065, USA. \\ Email: abou-alg@mskcc.org.
}

\begin{abstract}
Hepatoid adenocarcinoma of the stomach is an uncommon subtype of gastric cancer remarkably similar to hepatocellular carcinoma in histopathological analysis. It is also commonly associated with high serum alfa-fetoprotein and a poorer prognosis, despite the emergence of new therapeutic options. In recent years, next generation sequencing (NGS) technology has made it possible to identify and describe the genes and molecular alterations common to gastric cancer thereby contributing to the advancement of targeted therapies. A 62-year-old patient, with no prior risk factor for hepatocellular carcinoma (HCC), presented to the emergency room with dysphagia for solids, abdominal pain and weight loss of about 3 kilograms over 3 months. Histopathological analysis presented with disparities regarding HER2 and programmed deathligand 1 (PD-L1) status in the primary and metastatic sites. We describe a case of a de novo metastatic, human epidermal growth factor receptor 2 (HER2) positive esophagogastric junction hepatoid adenocarcinoma. Although this is a rare subgroup of gastric cancer, treatment strategies were based in recent studies in immunotherapy and guided therapy, taking into consideration the molecular findings from the patient's tumor NGS analysis. Data about HER2 and PDL1 heterogeneity were also reviewed. Despite the aggressiveness and rarity of this histology, the patient had a good response to treatment.
\end{abstract}

Keywords: Case report; hepatoid adenocarcinoma; esophagogastric junction; heterogeneity; human epidermal growth factor receptor 2 (HER2)

Submitted May 20, 2021. Accepted for publication Sep 30, 2021.

doi: 10.21037/jgo-21-287

View this article at: https://dx.doi.org/10.21037/jgo-21-287

\section{Introduction}

After the first description of alfa-fetoprotein (AFP) secreting gastrointestinal malignancies by McIntire et al. in 1975, Ishikura et al. described in 1985 seven gastric adenocarcinoma patients who presented high serum AFP and the first case of an hepatoid adenocarcinoma of the stomach (HAS). This uncommon subtype of gastric cancer is remarkably similar to hepatocellular carcinoma (HCC) in histopathological analysis and is frequently associated with poorer prognosis (1-4). Despite many theories about the origin of this histology, the exact mechanism is still not defined (5-7).

Besides the increased potential for liver metastasis, HAS is also associated with the production of AFP in the majority of cases, which is usually correlated with HCC (8). This protein is described as part of the immunohistochemical diagnostic of hepatoid adenocarcinoma, with other markers being glypican-3 (GPC-3), Sal-like protein 4 (SALL4) and 
less frequently Hepatocyte Paraffin 1 (Hep-Par 1) (9).

Despite the emergence of new therapeutic options for patients with esophagogastric cancer, the prognosis for metastatic and inoperable disease remains poor. Median overall survival (mOS) is approximately 1 year $(10,11)$. Performing next generation sequencing (NGS) and searching for targetable mutations in patients with metastatic esophagogastric cancer is thus crucial to identify predictive biomarkers of response (12). However, disparities regarding human epidermal growth factor receptor 2 (HER2) and programmed death-ligand 1 (PD-L1) status between the primary tumor and metastatic lesion have been described making, showing the heterogeneity of this markers in the systemic disease and making this scenario challenging (13).

Herein we describe a case of a patient presenting with a de novo metastatic hepatoid esophagogastric junction (EGJ) adenocarcinoma whose lesions displayed disparities in biomarkers analysis between the primary tumor in the stomach and metastatic site at the liver.

We present the following article in accordance with the CARE reporting checklist (available at https://dx.doi. org/10.21037/jgo-21-287).

\section{Case presentation}

A 62-year-old patient presented to the Emergency room with dysphagia for solids, abdominal pain and weight loss of about 3 kilograms over 3 months. He had a medical history of coronary disease and diabetes mellitus. Family oncologic history was significant for a father with gastric cancer at 55 -year-old, a brother with lung cancer at 48 -years-old and a mother with non-melanoma cutaneous cancer at 85 -year-old. No risk factors for HCC like history of alcohol use, known liver disease, or viral hepatitis were reported. A radiologic image screening of the abdomen was performed and showed evidence of diffuse tumor infiltration of the hepatic parenchyma. A liver tumor biopsy was obtained and showed invasive adenocarcinoma with tubular and hepatoid components, suggestive of a primary liver malignancy, including hepatocellular carcinoma (HCC). HER2 was positive (3+) and PD-L1 combined positive score (CPS) was 2 (Figure 1).

Staging was performed with whole body computed tomography (CT) that showed multiple confluent hepatic lesions with arterial phase enhancement and rapid wash out, accompanied by enlarged lymph nodes in left gastric, porta hepatis and portocaval chains (Figure 2). Despite this the rapid wash out being suggestive of HCC, this could note exclude hepatoid adenocarcinoma. Upper gastrointestinal endoscopy demonstrated a vegetative lesion surrounded by Barret epithelium in the distal esophagus (between 35 to $38 \mathrm{~cm}$ from the upper dental arcade). The histopathological analysis of this lesion described poorly cohesive cell carcinoma, HER2 negative and CPS of 12. Despite the presence of signet ring cells in this biopsy, a small part of the lesion was suggestive of HCC, corroborating the idea that this was the primary lesion with liver metastasis (Figure 3), as both biopsies showed areas of similar pathological findings (tubular hepatoid areas) and the patient had no HCC risk factors. The serum AFP at this moment was $20 \mathrm{ng} / \mathrm{mL}$.

Patient was already started on first line chemotherapy with docetaxel, oxaliplatin, leucovorin and 5-fluorouracil (FLOT) in another service. Therapy was discontinued though secondary to Common Terminology Criteria for Adverse Events (CTCAE) v 4.0 (14) grade three serum glutamic oxaloacetic transaminase elevation, grade three serum glutamic pyruvic transaminase elevation, grade two international normalized ratio (INR) elevation, grade one bilirubin elevation and grade four neutropenic colitis. At the resolution of these adverse effects and now the result of HER2 is reported, patient therapy was changed to 5 -fluorouracil, oxaliplatin and leucovorin (FOLFOX) plus trastuzumab in September 2019. Clinical response and decrease in AFP (from 19.568 to $48 \mathrm{ng} / \mathrm{mL}$ ) were noted after 3 cycles of therapy. By the fourth cycle of therapy, a radiologic RECIST 1.1 (15) partial response was noted (Figure 4). By the eleventh cycle of therapy, patient was switched to maintenance trastuzumab therapy (Figure 5).

Two months later, evident progression of disease and elevated AFP were noted. Therapy was changed to paclitaxel plus ramucirumab. Within three cycles of therapy, partial response was noted and was characterized by a decrease in the abdominal lymph nodes and hepatic metastasis size. This was followed by stable disease. However, due to peripheral neuropathy and colitis, paclitaxel was discontinued, and patient stayed on single agent ramucirumab with good tolerance. At this time, a NGS comprehensive panel from liver biopsy was performed and showed a low tumor mutational burden (TMB) - 2.52 mutations per megabase, along with CDK4 amplification, PIK3CA equivocal amplification, ERBB3 amplification, ETV6 deletion, SMAD4 splice site 1309-2A>G, TP53 Q192*, RNF43 splice site 953-276_998>AC. Microsatellite status (MS) was stable and there were no FGFR alterations.

All procedures performed in studies involving human 

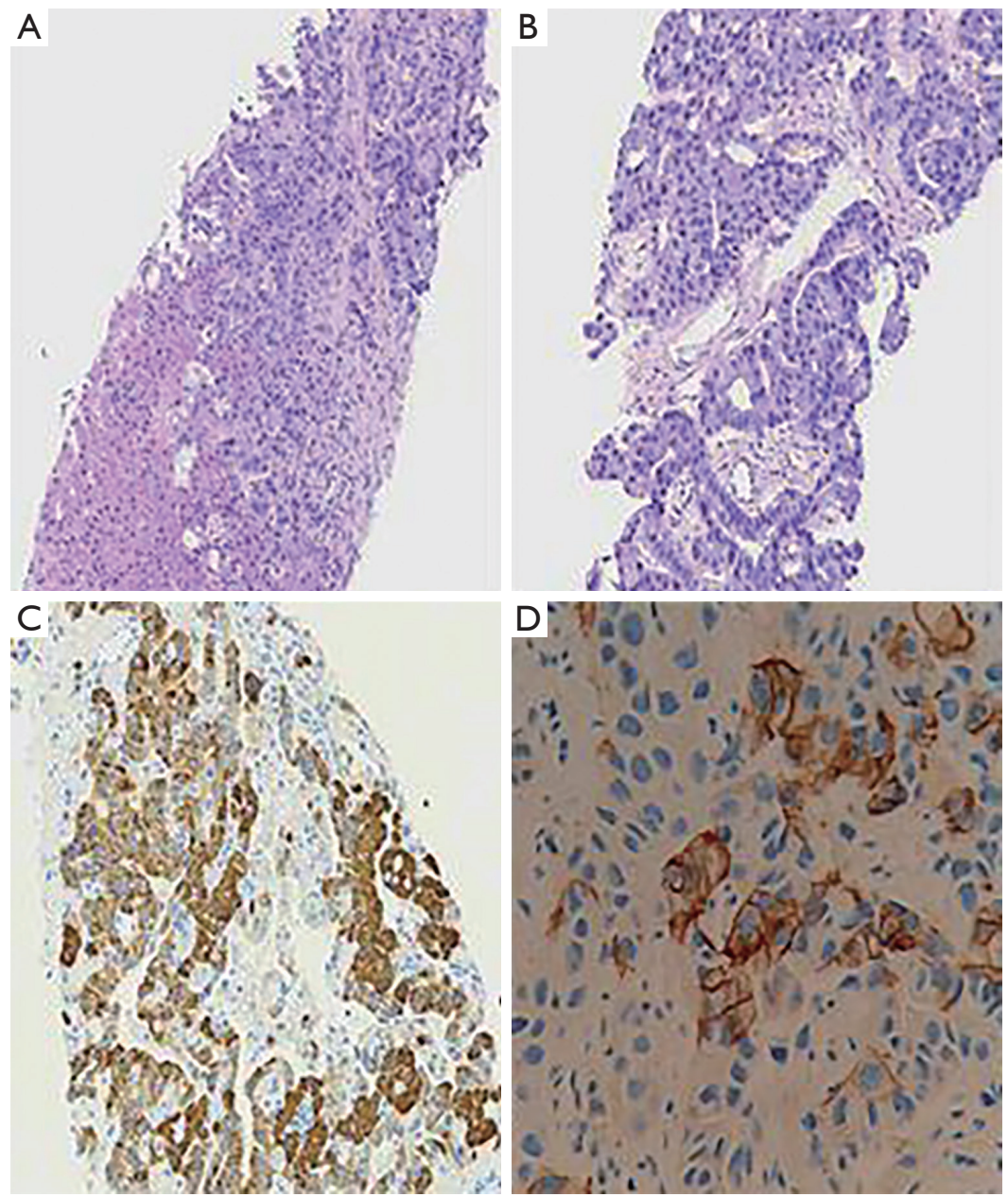

Figure 1 Pathology high magnification views. Histopathological tissue analysis from liver biopsy revealed a tubular and hepatoid adenocarcinoma $(A, B ; \times 100)$. Immunohistochemistry stained positive for Hepatocyte $(C ; \times 100)$ and HER2 3+ (D; ×400).
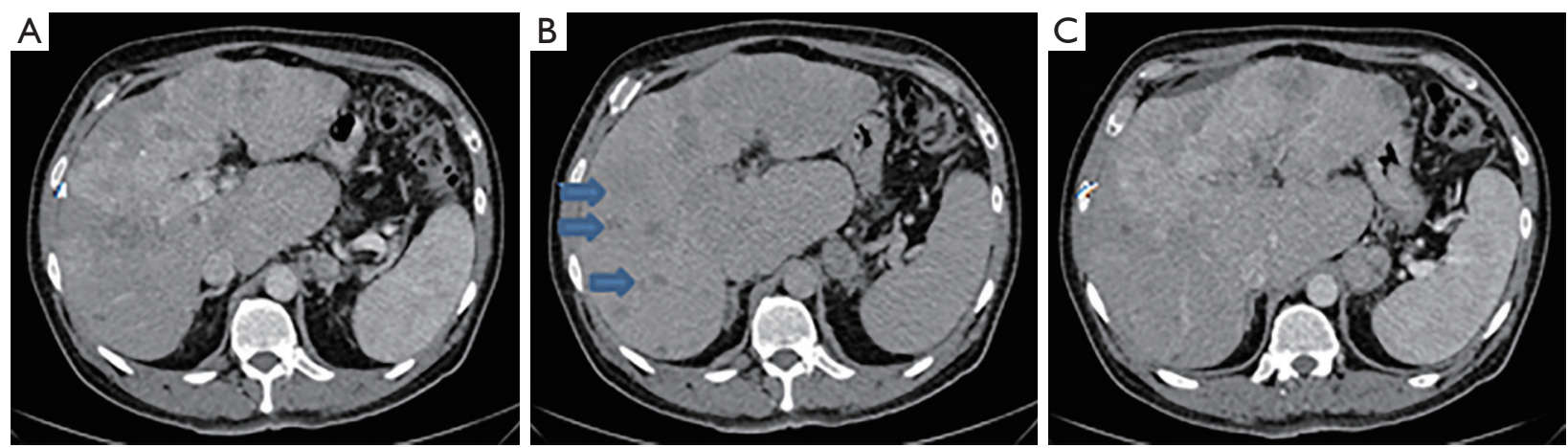

Figure 2 CT scan liver views. Chronic liver disease (liver enlargement and splenomegaly). Several liver lesions with early arterial phase enhancement (A) and rapid washout (B, blue arrows), suspicious for hepatocellular carcinoma. Enlarged gastrohepatic node (C). 

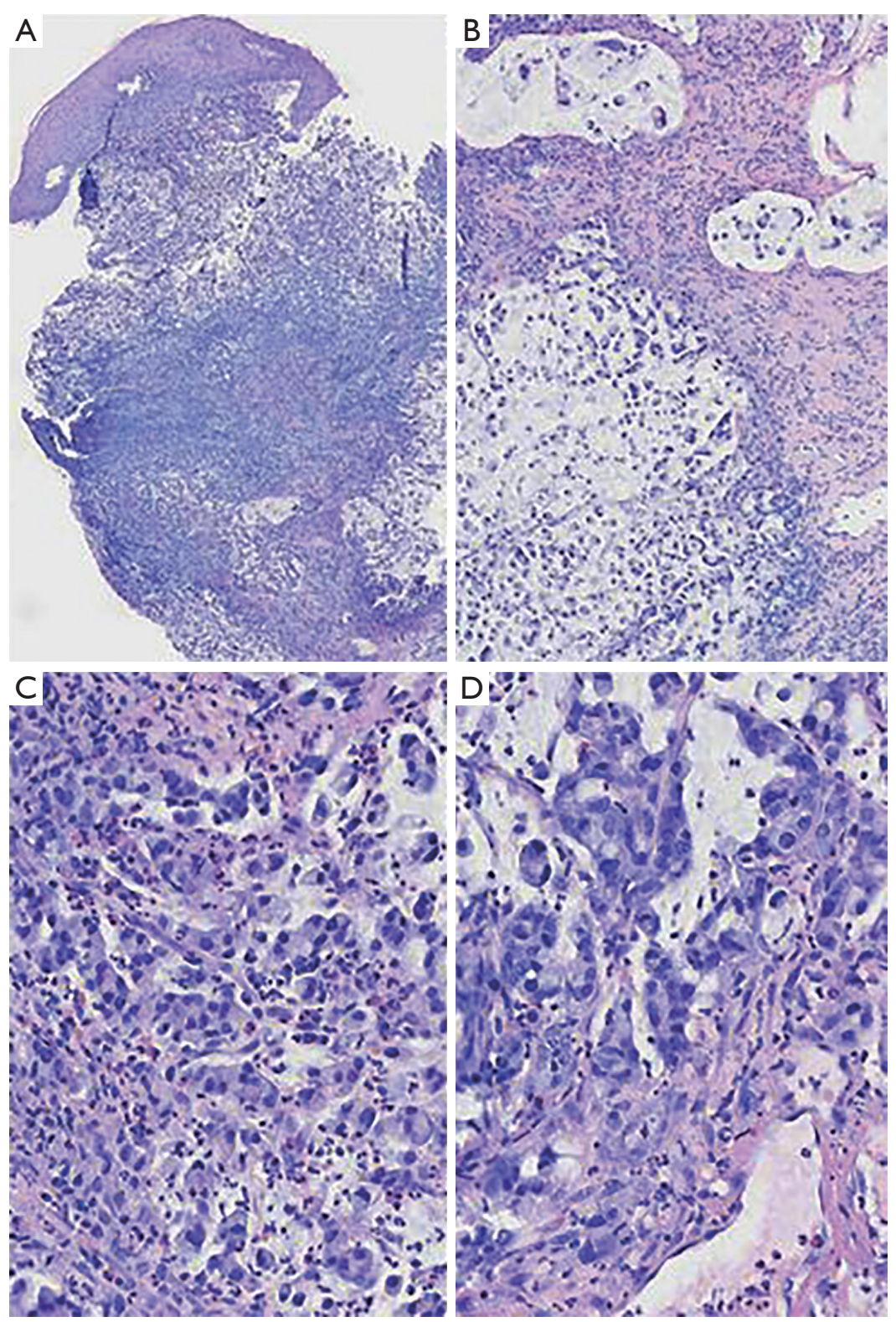

Figure 3 Pathology high and low magnification. Histopathological tissue analysis from esophagogastric junction biopsy revealed a poorly cohesive adenocarcinoma, signet-ring type $(A, B ; \times 100)$. There was also a focal tubular and hepatoid features $(C, D ; \times 400)$.

participants were in accordance with the ethical standards of the institutional and/or national research committee(s) and with the Declaration of Helsinki (as revised in 2013). Informed consent was obtained from the patient.

\section{Discussion}

The incorporation of NGS technology in recent studies has allowed the development of comprehensive datasets that made possible the description of many genetic characteristics of gastric cancer (16-18). Notably, the TCGA Research network defined four major genomic subtypes of gastric cancer based on genetic profile (16). However, due to the rarity and the erratic geographic distribution, genetic alterations in HAS could not be found in a retrospective analysis of this database (19).

Wang et al. performed an NGS panel of 483 cancerrelated genes on a population of 23 HAS and 18 clinical 

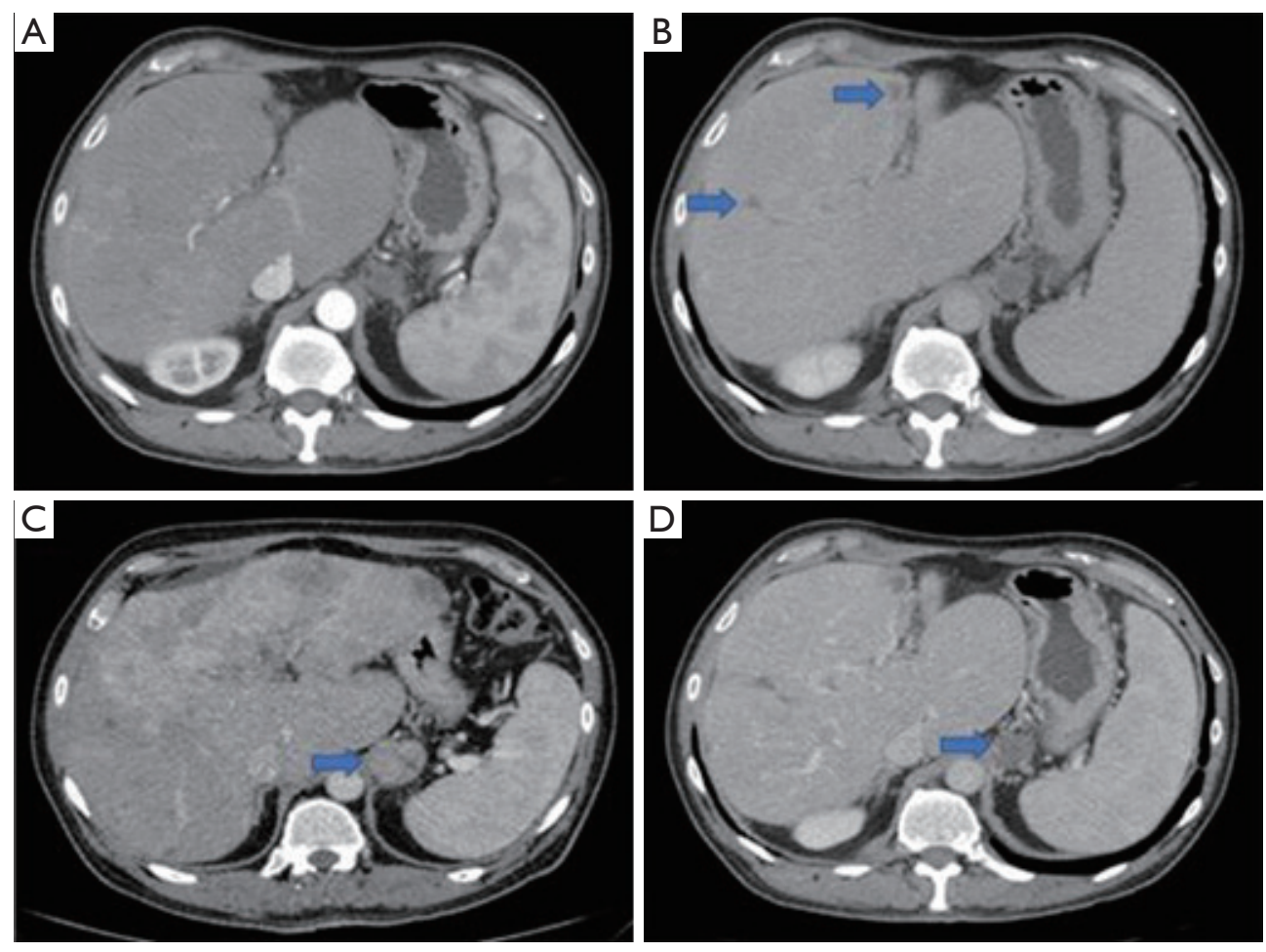

Figure 4 Liver lesion and lymph node regression after first line chemotherapy with FOLFOX and trastuzumab. Nearly homogeneous enhancement of the liver with disappearance of hypervascular areas of enhancement on arterial phase (A) and just a few residual areas of washout on the portal venous phase (B). Previously enlarged gastrohepatic node (C, blue arrow) has decreased in size and attenuation (D, blue arrow).

parameter-matched common gastric cancer (CGC) (20). They found that the most frequent mutated gene was TP53, consistent with previous reports and the TCGA database. However, they found a higher frequency of mutations in CEBPA, RPTOR, WISP3, MARK1 and CD3EAP (10-20\%) (16,20,21). Copy number variant (CNV), usually correlated with the overexpression of cancer-promoting driver genes (22), was also analyzed and tended to occur more commonly in HAS than CGC, especially in the genes TOP1 (50\%), STK4 (45.5\%), CDKN1B (40.9\%), H3F3A (36.4\%), MYC (22.7\%), CCNE1 (22.7\%), NFKBIA (22.7\%), VEGFA (18.2\%), CCND3 (13.6\%) and $\mathrm{E} 2 \mathrm{~F} 1(13.6 \%)$. Besides that, and based on the analysis of mutations and copy number gains (CNGs), the authors also found that several pathways were significantly enriched in both HAS and CGC (ErbB, PI3K-Akt and the p53 signaling pathway). HIF-1 signaling pathway and the signaling pathway regulating the pluripotency of stem cells thought were especially enriched in HAS. These findings could guide novel personalized therapies going forward (20).
In the first-line setting, taking into consideration the HER2 positivity on the liver metastasis and the results from the TOGA trial (absolute benefit of approximately 4 months in overall survival (OS) for advanced HER2 positive gastric and EGJ cancers) (23), it was decided to initiate trastuzumab plus chemotherapy followed by maintenance trastuzumab. Many studies have proven that the frequency of overexpression of HER2 is slightly higher for EGJ in comparison to the stomach (23-25). Also, when compared to breast cancer, the heterogeneity of this analysis is higher in gastroesophageal adenocarcinoma, with differences in immunostaining requirements between these two neoplasms (26-28).

Nevertheless, the clinical impact of HER2 heterogeneity has already been described not only in breast cancer but also in gastric cancer $(29,30)$. In a trial with twenty-eight HER2 positive gastric cancer treated with gastrectomy and trastuzumab-based chemotherapy, Wakatsuki et al. suggested that intratumoral HER2 heterogeneity may have a robust impact on trastuzumab efficacy in this population. 


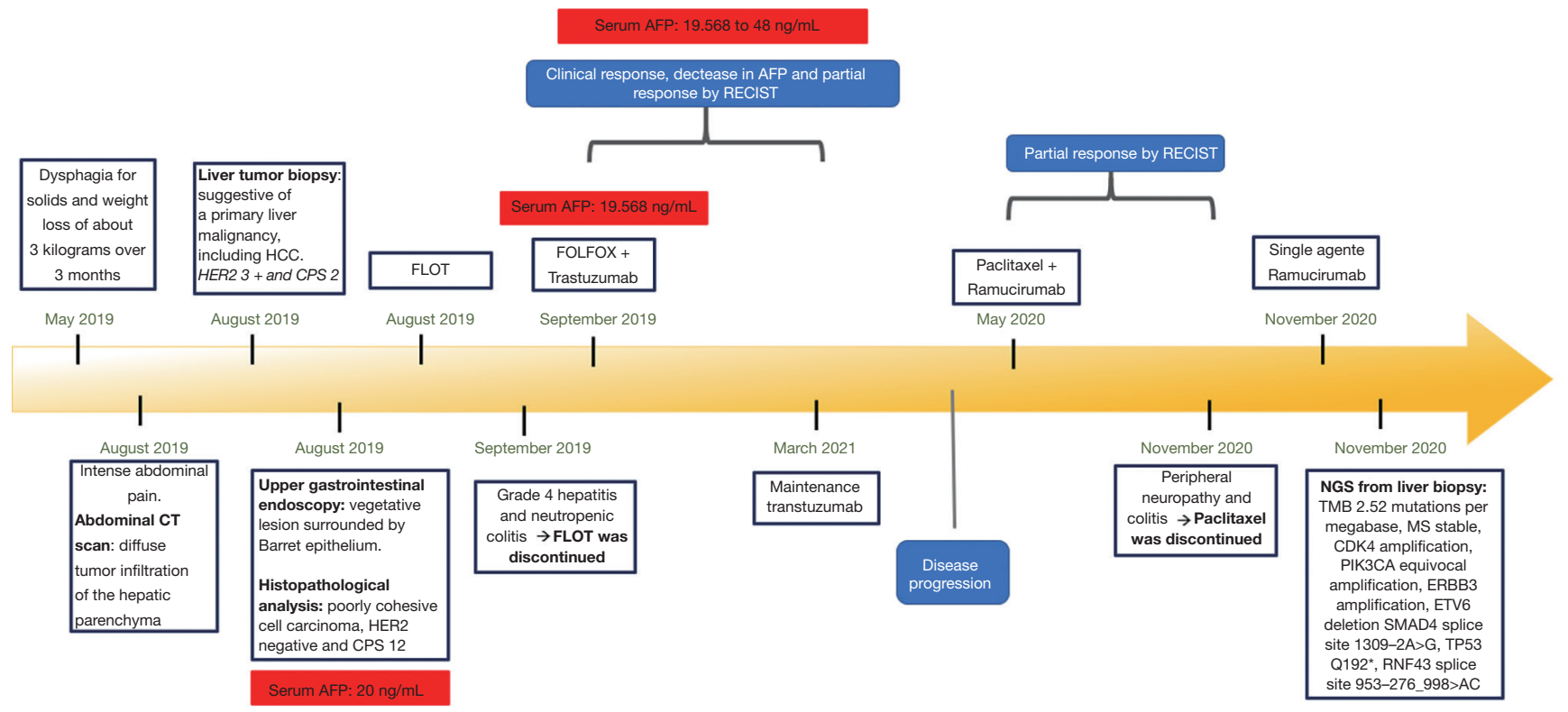

Figure 5 Timeline of the case report. AFP, alfa-fetoprotein; CT, computed tomography; FLOT, docetaxel, oxaliplatin, leucovorin and 5-fluoracil; FOLFOX, 5 -fluoruracil, oxaliplatin and leucovorin; RECIST, Response Evaluation Criteria in Solid Tumours.

Patients defined as homo HER2-positive group had significantly longer progression-free survival (PFS) than the hetero HER2-positive group (20 vs. 6 months, HR 0.11, $\mathrm{P}<0.001$ ), with an additional benefit for OS (not reached vs. 14 months, HR 0.18, $\mathrm{P}=0.003$, respectively) (30). More recently, Butter et al. concluded that predicted HER2 assessment based on biopsies of these neoplasms can lead to false-negative results, with the possibility of HER2 positive tumors being denied of neoadjuvant HER2 therapy. These findings were based on the analysis of 378 adenocarcinomas of the esophagus or stomach paired biopsies and resection specimens (31).

Also, discordant HER2 analysis between primary and metastatic tumors is already described but is a rare event. A meta-analysis conducted by Peng et al. focused on this scenario (spatial HER2 heterogeneity) in gastric cancers and found that only $7 \%$ of cases (95\% CI: $5-10 \%$ ) had HER2 discordance (32).

Besides the results in first-line, the anti-HER2 blockade in subsequent lines historically did not present relevant benefit, with lack of improvement in OS in the TyTAN (lapatinib plus paclitaxel) and GATSBY (trastuzumab emtansine) trials $(33,34)$. Even so, more recently the DESTINY Gastric01 trial proved that trastuzumab deruxtecan led to significant improvement in OS and overall response rate in patients that progressed on two or more prior regimens, presenting as a new option in the future for patients in advanced line settings (35).

Our patient also presented with discordant HER 2 status in immunohistochemistry (IHC) and NGS. Janjigian et al. reported a strong correlation between ERBB2 copy number as determined by sequencing (pretreatment) and HER2 IHC/ FISH in esophagogastric cancer, with a concordance rate of $93.7 \%$ (36). Similarly to our patient, their 4 discordant cases presented with significantly shorter PFS on first-line trastuzumab (median PFS 5.8 vs. 14 months). The authors concluded that the intrinsic and acquired resistance to anti$H E R 2$ therapy was also related to molecular alterations such as lack of $E R B B 2$ amplification in NGS, deletion of ERBB2 exon 16, and commutations in the receptor tyrosine kinase, RAS, and PI3K pathways, with this last one also amplified in our case.

Ramucirumab, a monoclonal antibody against vascular endothelial growth factor receptor 2 (VEGFR-2), is widely approved for second-line treatment in adults with advanced gastric or GEJ adenocarcinoma, as monotherapy or combined with paclitaxel, based on two phase III trials (REGARD and RAINBOW) (37-39). However, there are limited data on potential biomarkers of response to ramucirumab and others antiangiogenic agents in gastric cancer and GEJ scenario, especially in second-line (40). The decision of initiating ramucirumab plus paclitaxel was supported on the similarity 
already described between HAS and HCC, alongside the results from REACH and REACH 2 trials $(41,42)$. In a subgroup analysis of the REACH trial, which evaluated ramucirumab versus placebo in the second-line treatment in patients with advanced HCC, improvement in overall survival was noted in the population with a baseline concentration of alfa-fetoprotein of $400 \mathrm{ng} / \mathrm{mL}$ or greater (41). Based on this information, REACH 2 selected only patients with advanced HCC and increased alfa-fetoprotein of $400 \mathrm{ng} / \mathrm{mL}$ or greater and treated them with ramucirumab or placebo in the second line after sorafenib. The primary endpoint of OS was reached (mOS 8.5 vs. 7.3 months, HR $0.710, \mathrm{P}=0.0199)$. While these results are suggestive of alfa fetoprotein as a biomarker for response with Ramucirumab in the HCC population, this claim remains debatable $(42,43)$.

In recent years, immunotherapy is a growing option for the treatment of gastric and EGJ, especially in the metastatic and advanced scenario. The first results from CHECKMATE 649 demonstrated that nivolumab was the first anti-Programmed Cell Death Protein (PD1) inhibitor with benefit in OS and PFS when combined with chemotherapy (versus chemotherapy alone), in previously untreated advanced and metastatic HER2 negative esophageal, GC and EGJ adenocarcinoma. Besides the OS benefit being significant in Combined Positive Score (CPS) $\geq 1$, the primary endpoint was achieved in tumors that expressed PD-L1 CPS $\geq 5$ (OS, HR 0.71, P<0.0001) (44).

In KEYNOTE 062, pembrolizumab alone or combined with chemotherapy was not superior to chemotherapy alone for the PFS and OS endpoints (45). There is no clear answer to this discrepancy, however, there have been many discussions about tumor spatial and temporal heterogeneity of PD-L1 and TMB when obtaining tumor samples for molecular testing. This phenomenon was exemplified by Zhou et al. in gastroesophageal adenocarcinoma when they compared these two biomarkers at baseline diagnosis and after chemotherapy (46). It was concluded that both exhibited marked spatial and temporal heterogeneity, characteristic that should be considered when deciding the best immunotherapy treatment.

Based on the knowledge that adding trastuzumab in the first line of HER2-positive metastatic gastric cancer improved overall survival, the combination of trastuzumab, pembrolizumab and chemotherapy was tested in HER2positive GC, esophageal and EGJ adenocarcinoma in a phase II trial. The primary endpoint was achieved and $70 \%$ of 37 patients were progression-free at 6 months, with a safety profile of adverse events and a promising activity of the combination to be confirmed in phase III studies (47).

\section{Conclusions}

We report a case of a de novo metastatic hepatoid adenocarcinoma of esophagogastric junction whose lesions displayed disparities between primary tumor and metastatic site regarding predictive biomarkers. The patient was treated with trastuzumab plus chemotherapy in the first line and paclitaxel and ramucirumab in the second line. Tumor heterogeneity is a well-described phenomenon that interferes in biomarkers of treatment response, but despite the aggressiveness and rarity of this histology, the patient had a good response to treatment. Novel and composite treatment protocols, as well as reliable biomarkers of response, are needed to improve the survival of these patients.

\section{Acknowledgments}

This case was presented at the Memorial Sloan Kettering Global Fellows Gastrointestinal Oncology conference on November 11, 2020.

Funding: The effort was supported by the endowment gift of Mrs. Mamdouha El-Sayed Bobst and the Bobst Foundation. The effort was also funded by Hospital Sírio Libanês.

\section{Footnote}

Provenance and Peer Review: This article was commissioned by the editorial office, Fournal of Gastrointestinal Oncology for the series "Educational Case Series of the Memorial Sloan Kettering Cancer Center". The article has undergone external peer review.

Reporting Checklist: The authors have completed the CARE reporting checklist. Available at https://dx.doi. org/10.21037/jgo-21-287

Peer Review File: Available at https://dx.doi.org/10.21037/ jgo-21-287

Conflicts of Interest: The authors have completed the ICMJE uniform disclosure form (available at https:// dx.doi.org/10.21037/jgo-21-287). The series "Educational Case Series of the Memorial Sloan Kettering Cancer Center" was commissioned by the editorial office without any sponsorship or funding. TBC received support from 
Astra Zeneca, Bayer, Eli Lilly, Genentech/Roche, Ipsen, Merck KGaA, Merck Sharp \& Dohme. AS received support from Roche, Merck, BMS, Sanofi, Eli Lilly and is a consultant to Roche, MSD, Merck, Sanofi, Astellas and Johnson \& Johnson, and he received honoraria for lectures and presentation from Roche, Merck, BMS, MSD, Johnson \& Johnson and Amgen and support for attending meetings from Merck. GMB receives research funding to his institution from mAbxience, Merck Sharp \& Dohme Corp. and Bristol-Myers Squibb. DM received research support from Astellas, Pfizer, and Astra Zeneca, and honoraria/travel support from MSD, BMS, Astellas, Bayer and Janssen. GdSF received honoraria/travel support from BMS, MSD, Roche and Ipsen and advisory board support from BMS, MSD, Boeringher, Sanofi, Roche, Astelas, Bayer. GKA served as the unpaid Guest Editor for the series and he received research support from Arcus, Agios, Astra Zeneca, BioNtech, BMS, Celgene, Flatiron, Genentech/Roche, Genoscience, Incyte, Polaris, Puma, QED, Silenseed, Yiviva, and consulting support for efforts with Adicet, Astra Zeneca, Alnylam, Autem, Bayer, Beigene, Berry Genomics, Cend, Celgene, CytomX, Eisai, Eli Lilly, Exelixis, Flatiron, Genentech/Roche, Genoscience, Helio, Incyte, Ipsen, Legend Biotech, Merck, Nerviano, QED, Redhill, Rafael, Servier, Silenseed, Sillajen, Sobi, Surface Oncology, Therabionics, Vector, and Yiviva. YYJ received research support from Bayer, Bristol-Myers Squibb, Cycle for Survival, Department of Defense, Eli Lilly, Fred's Team, Genentech/Roche, Merck, NCI, RGENIX and receives consulting fees from Astra Zeneca, Basilea Pharmaceutica, Bayer, Bristol-Myers Squibb, Daiichi-Sankyo, Eli Lilly, Imugene, Merck, Merck Serono, Michael J. Hennessy Associates, Paradigm Medical Communications, Pfizer, RGENIX, Seagen, Zymeworks Inc., and has Stock Options of RGENIX. The authors have no other conflict of interest to report.

Ethical Statement: The authors are accountable for all aspects of the work in ensuring that questions related to the accuracy or integrity of any part of the work are appropriately investigated and resolved. All procedures performed in studies involving human participants were in accordance with the ethical standards of the institutional and/or national research committee(s) and with the Declaration of Helsinki (as revised in 2013). Written informed consent was obtained from the patient for publication of this case report and images referring to it. A copy of the written consent is available for review by the editorial office of this journal.

Open Access Statement: This is an Open Access article distributed in accordance with the Creative Commons Attribution-NonCommercial-NoDerivs 4.0 International License (CC BY-NC-ND 4.0), which permits the noncommercial replication and distribution of the article with the strict proviso that no changes or edits are made and the original work is properly cited (including links to both the formal publication through the relevant DOI and the license). See: https://creativecommons.org/licenses/by-nc-nd/4.0/.

\section{References}

1. Liu X, Cheng Y, Sheng W, et al. Analysis of clinicopathologic features and prognostic factors in hepatoid adenocarcinoma of the stomach. Am J Surg Pathol 2010;34:1465-71.

2. Lin CY, Yeh HC, Hsu CM, et al. Clinicopathologial features of gastric hepatoid adenocarcinoma. Biomed J 2015;38:65-9.

3. Qu BG, Bi WM, Qu BT, et al. PRISMA-Compliant Article: Clinical Characteristics and Factors Influencing Prognosis of Patients With Hepatoid Adenocarcinoma of the Stomach in China. Medicine (Baltimore) 2016;95:e3399.

4. Yano T, Kishimoto T, Tomaru U, et al. Further evidence of hepatic transdifferentiation in hepatoid adenocarcinomas of the stomach: quantitative analysis of mRNA for albumin and hepatocyte nuclear factor-4alpha. Pathology 2003;35:75-8.

5. Fujii H, Ichikawa K, Takagaki T, et al. Genetic evolution of alpha fetoprotein producing gastric cancer. J Clin Pathol 2003;56:942-9.

6. Akiyama S, Tamura G, Endoh Y, et al. Histogenesis of hepatoid adenocarcinoma of the stomach: molecular evidence of identical origin with coexistent tubular adenocarcinoma. Int J Cancer 2003;106:510-5.

7. Bialecki ES, Di Bisceglie AM. Diagnosis of hepatocellular carcinoma. HPB (Oxford) 2005;7:26-34.

8. Ushiku T, Shinozaki A, Shibahara J, et al. SALL4 represents fetal gut differentiation of gastric cancer, and is diagnostically useful in distinguishing hepatoid gastric carcinoma from hepatocellular carcinoma. Am J Surg Pathol 2010;34:533-40.

9. Zhao M, Sun L, Lai JZ, et al. Expression of RNA-binding protein LIN28 in classic gastric hepatoid carcinomas, gastric fetal type gastrointestinal adenocarcinomas, and 
hepatocellular carcinomas: An immunohistochemical study with comparison to SALL4, alpha-fetoprotein, glypican-3, and Hep Par1. Pathol Res Pract 2018;214:1707-12.

10. Cunningham D, Starling N, Rao S, et al. Capecitabine and oxaliplatin for advanced esophagogastric cancer. N Engl J Med 2008;358:36-46.

11. Van Cutsem E, Moiseyenko VM, Tjulandin S, et al. Phase III study of docetaxel and cisplatin plus fluorouracil compared with cisplatin and fluorouracil as first-line therapy for advanced gastric cancer: a report of the V325 Study Group. J Clin Oncol 2006;24:4991-7.

12. Zehir A, Benayed R, Shah RH, et al. Mutational landscape of metastatic cancer revealed from prospective clinical sequencing of 10,000 patients. Nat Med 2017;23:703-13.

13. Zou Y, Hu X, Zheng S, Yang A, Li X, Tang H, et al. Discordance of immunotherapy response predictive biomarkers between primary lesions and paired metastases in tumours: A multidimensional analysis. EBioMedicine 2021;63:103137.

14. National Cancer Institute (U.S.). Common terminology criteria for adverse events: (CTCAE) version 4. [Bethesda, Md.]: U.S. Department of Health and Human Services; 2010.

15. Eisenhauer EA, Therasse P, Bogaerts J, et al. New response evaluation criteria in solid tumours: revised RECIST guideline (version 1.1). Eur J Cancer 2009;45:228-47.

16. Cancer Genome Atlas Research Network. Comprehensive molecular characterization of gastric adenocarcinoma. Nature 2014;513:202-9.

17. Cristescu R, Lee J, Nebozhyn M, et al. Molecular analysis of gastric cancer identifies subtypes associated with distinct clinical outcomes. Nat Med 2015;21:449-56.

18. Ye XS, Yu C, Aggarwal A, et al. Genomic alterations and molecular subtypes of gastric cancers in Asians. Chin J Cancer 2016;35:42.

19. Arora K, Bal M, Shih A, et al. Fetal-type gastrointestinal adenocarcinoma: a morphologically distinct entity with unfavourable prognosis. J Clin Pathol 2018;71:221-7.

20. Wang Y, Sun L, Li Z, et al. Hepatoid adenocarcinoma of the stomach: a unique subgroup with distinct clinicopathological and molecular features. Gastric Cancer 2019;22:1183-92.

21. Akazawa Y, Saito T, Hayashi T, et al. Next-generation sequencing analysis for gastric adenocarcinoma with enteroblastic differentiation: emphasis on the relationship with hepatoid adenocarcinoma. Hum Pathol 2018;78:79-88.

22. Liang L, Fang JY, Xu J. Gastric cancer and gene copy number variation: emerging cancer drivers for targeted therapy. Oncogene 2016;35:1475-82.

23. Bang YJ, Van Cutsem E, Feyereislova A, et al. Trastuzumab in combination with chemotherapy versus chemotherapy alone for treatment of HER2-positive advanced gastric or gastro-oesophageal junction cancer (ToGA): a phase 3, open-label, randomised controlled trial. Lancet 2010;376:687-97.

24. Van Cutsem E, Bang YJ, Feng-Yi F, et al. HER2 screening data from ToGA: targeting HER2 in gastric and gastroesophageal junction cancer. Gastric Cancer 2015;18:476-84.

25. Ieni A, Barresi V, Giuffrè G, et al. HER2 status in advanced gastric carcinoma: A retrospective multicentric analysis from Sicily. Oncol Lett 2013;6:1591-4.

26. Albarello L, Pecciarini L, Doglioni C. HER2 testing in gastric cancer. Adv Anat Pathol 2011;18:53-9.

27. Pirrelli M, Caruso ML, Di Maggio M, et al. Are biopsy specimens predictive of HER2 status in gastric cancer patients? Dig Dis Sci 2013;58:397-404.

28. Hofmann M, Stoss O, Shi D, et al. Assessment of a HER2 scoring system for gastric cancer: results from a validation study. Histopathology 2008;52:797-805.

29. Lee HJ, Seo AN, Kim EJ, et al. HER2 heterogeneity affects trastuzumab responses and survival in patients with HER2-positive metastatic breast cancer. Am J Clin Pathol 2014;142:755-66.

30. Wakatsuki T, Yamamoto N, Sano T, et al. Clinical impact of intratumoral HER2 heterogeneity on trastuzumab efficacy in patients with HER2-positive gastric cancer. J Gastroenterol 2018;53:1186-95.

31. Butter R, Hooijer G, van der Wel M, et al. HER2 heterogeneity in adenocarcinoma of the distal esophagus and stomach. J Clin Oncol 2020;38:4567.

32. Peng Z, Zou J, Zhang X, et al. HER2 discordance between paired primary gastric cancer and metastasis: a metaanalysis. Chin J Cancer Res 2015;27:163-71.

33. Satoh T, Xu RH, Chung HC, et al. Lapatinib plus paclitaxel versus paclitaxel alone in the second-line treatment of HER2-amplified advanced gastric cancer in Asian populations: TyTAN--a randomized, phase III study. J Clin Oncol 2014;32:2039-49.

34. Thuss-Patience PC, Shah MA, Ohtsu A, et al. Trastuzumab emtansine versus taxane use for previously treated HER2positive locally advanced or metastatic gastric or gastrooesophageal junction adenocarcinoma (GATSBY): an international randomised, open-label, adaptive, phase 2/3 study. Lancet Oncol 2017;18:640-53.

35. Shitara K, Bang YJ, Iwasa S, et al. Trastuzumab 
Deruxtecan in Previously Treated HER2-Positive Gastric

Cancer. N Engl J Med 2020;382:2419-30.

36. Janjigian YY, Sanchez-Vega F, Jonsson P, et al. Genetic Predictors of Response to Systemic Therapy in Esophagogastric Cancer. Cancer Discov 2018;8:49-58.

37. Greig SL, Keating GM. Ramucirumab: A Review in Advanced Gastric Cancer. BioDrugs 2015;29:341-51.

38. Fuchs CS, Tomasek J, Yong CJ, et al. Ramucirumab monotherapy for previously treated advanced gastric or gastro-oesophageal junction adenocarcinoma (REGARD): an international, randomised, multicentre, placebocontrolled, phase 3 trial. Lancet 2014;383:31-9.

39. Wilke H, Muro K, Van Cutsem E, et al. Ramucirumab plus paclitaxel versus placebo plus paclitaxel in patients with previously treated advanced gastric or gastro-oesophageal junction adenocarcinoma (RAINBOW): a double-blind, randomised phase 3 trial. Lancet Oncol 2014;15:1224-35.

40. Young K, Smyth E, Chau I. Ramucirumab for advanced gastric cancer or gastro-oesophageal junction adenocarcinoma. Therap Adv Gastroenterol 2015;8:373-83.

41. Zhu AX, Park JO, Ryoo BY, et al. Ramucirumab versus placebo as second-line treatment in patients with advanced hepatocellular carcinoma following first-line therapy with sorafenib (REACH): a randomised, double-blind, multicentre, phase 3 trial. Lancet Oncol 2015;16:859-70.

42. Zhu AX, Kang YK, Yen CJ, et al. Ramucirumab after sorafenib in patients with advanced hepatocellular carcinoma and increased $\alpha$-fetoprotein concentrations

Cite this article as: de Castria TB, Tang L, Queiroz MM, Awni BM, Paroder V, Shamseddine A, Bariani GM, Mukherji D, Matar CF, Fernandes GS, El-Olayan A, Sabatin F, Elias R, Gupta R, Janjigian YY, Abou-Alfa GK. Hepatoid esophagogastric adenocarcinoma and tumoral heterogeneity: a case report. J Gastrointest Oncol 2021;12(6):3123-3132. doi: 10.21037/jgo-21287
(REACH-2): a randomised, double-blind, placebocontrolled, phase 3 trial. Lancet Oncol 2019;20:282-96.

43. Abou-Alfa GK. Ramucirumab and the controversial role of $\alpha$-fetoprotein in hepatocellular carcinoma. Lancet Oncol 2019;20:177-9.

44. Janjigian YY, Shitara K, Moehler M, et al. First-line nivolumab plus chemotherapy versus chemotherapy alone for advanced gastric, gastro-oesophageal junction, and oesophageal adenocarcinoma (CheckMate 649): a randomised, open-label, phase 3 trial. Lancet 2021;398:27-40.

45. Shitara K, Van Cutsem E, Bang YJ, et al. Efficacy and Safety of Pembrolizumab or Pembrolizumab Plus Chemotherapy vs Chemotherapy Alone for Patients With First-line, Advanced Gastric Cancer: The KEYNOTE-062 Phase 3 Randomized Clinical Trial. JAMA Oncol 2020;6:1571-80.

46. Zhou KI, Peterson B, Serritella A, et al. Spatial and Temporal Heterogeneity of PD-L1 Expression and Tumor Mutational Burden in Gastroesophageal Adenocarcinoma at Baseline Diagnosis and after Chemotherapy. Clin Cancer Res 2020;26:6453-63.

47. Janjigian YY, Maron SB, Chatila WK, et al. First-line pembrolizumab and trastuzumab in HER2-positive oesophageal, gastric, or gastro-oesophageal junction cancer: an open-label, single-arm, phase 2 trial. Lancet Oncol 2020;21:821-31. 